Utah State University

DigitalCommons@USU

$1-1-2007$

\title{
Statistical Approach to Validation of Satellite Atmospheric Retrievals
}

Nikita Pougatchev

Gail Bingham

Dian Seidel

Franz Berger

Follow this and additional works at: https://digitalcommons.usu.edu/sdl_pubs

\section{Recommended Citation}

Pougatchev, Nikita; Bingham, Gail; Seidel, Dian; and Berger, Franz, "Statistical Approach to Validation of Satellite Atmospheric Retrievals" (2007). Space Dynamics Lab Publications. Paper 108.

https://digitalcommons.usu.edu/sdl_pubs/108

This Article is brought to you for free and open access by the Space Dynamics Lab at DigitalCommons@USU. It has been accepted for inclusion in Space Dynamics Lab Publications by an authorized administrator of DigitalCommons@USU. For more information, please contact digitalcommons@usu.edu.

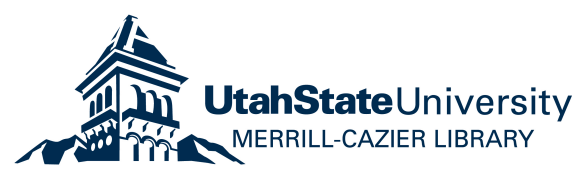




\title{
Statistical Approach to Validation of Satellite Atmospheric Retrievals
}

\author{
Nikita. Pougatchev $^{1 \mathrm{a}}$, Gail Bingham ${ }^{\mathrm{a}}$, Dian Seidel ${ }^{\mathrm{b}}$, and Franz Berger ${ }^{\mathrm{c}}$ \\ a Space Dynamics Laboratory, 1695 North Research Park Way North Logan, UT 84341; \\ b NOAA Air Resources Laboratory (R/ARL), 1315 East West Highway, \\ Silver Spring, MD 20910; \\ ${ }^{c}$ German Meteorological Service, Meteorological Observatory Lindenberg, \\ Richard-Aßmann-Observatory (MOL-RAO), Am Observatorium 12, \\ D-15848 Tauche / Lindenberg;
}

\begin{abstract}
A mathematical model for statistical estimate of the bias and noise in satellite retrievals of atmospheric profiles and a case study are presented. The model allows accurate validation of actual performance of the remote sensing system while in orbit by comparing its measurements to correlative data sets, e. g. radiosonde network. The model accounts for the following factors: (i) The satellite and validating systems sample volumes of the atmosphere at times and locations that are not exactly co-located. (ii) The validated and validating systems have different characteristics, e. g. different vertical resolution and noise level. All the above factors cause apparent difference between the data to be compared. The presented model makes the comparison accurate by allowing for the differences. To demonstrate its practicability we present the case study that involves the radiosonde data from three stations: ARM Tropical Western Pacific $\left(0.5^{\circ} \mathrm{S}, 167^{\circ}\right.$ E), ARM Southern Great Planes $\left(37^{\circ} \mathrm{N}, 98^{\mathrm{O}} \mathrm{W}\right)$, and Lindenberg $\left(52^{\mathrm{O}} \mathrm{N}, 14^{\mathrm{O}} \mathrm{E}\right)$. For each station we considered temperature profile validation scenario and estimated associated errors. The model can be used for interpretation of the validation results when the above mentioned sources of discrepancies are significant, as well as for evaluation of validation data sources, e.g. GRUAN (GCOS Reference Upper-Air Network).
\end{abstract}

Keywords: Atmospheric, Validation, Remote sensing, Retrieval, Radiosonde network

\section{INTRODUCTION}

Atmospheric sounders, i. e. instruments remotely measuring atmospheric thermodynamic parameters and constituents are important source of data for numerous practical and scientific applications. They compose the key component of the Global Earth Observation System of Systems (GEOSS) ${ }^{1}$. To be usable the data from satellite sounders must be validated in a sense that their relation to the true state of the atmosphere is known with statistically estimated error. Thus, we define the validation as an activity whose purpose is to estimate the error of the sounder during its operation.

In the context of current work the term atmospheric sounder implies satellite-borne instrument, but the presented validation methodology and practical technique can be applied to any remote sensing system which needs to be validated. In the process of designing, pre-launch testing, and calibration of a measurement system nominal relations between the true state and measurement results are established. Following Clive Rodgers ${ }^{2}$ we call this characterization and error analysis. After launch the actual errors may differ from the errors established during pre-launch analysis. That may be caused by changes in the instrument performance, by inaccuracy in atmospheric radiative transfer modeling, etc. Thus, special efforts should be made to validate the sounding results during the in-orbit phase of the satellite system.

One of the ways to do the validation is to perform a proper comparison of satellite data with an independently acquired reference data set. The reference system can be in situ air-borne, e. g. radiosondes $^{3,4}$, air-borne remote ${ }^{5}$, or space-borne ${ }^{6}$. Because a remote sounder measures some function of the atmosphere-surface state ${ }^{2}$, the ideal validation would be

$\overline{1}$ nikita.pougatchev@sdl.usu.edu; tel. +1 435 797-4042

Remote Sensing of Clouds and the Atmosphere XII,

edited by Adolfo Comerón, Richard H. Picard, Klaus Schäfer, James R. Slusser, Aldo Amodeo,

Proc. of SPIE Vol. 6745, 674511, (2007) · 0277-786X/07/\$18 - doi: 10.1117/12.737943

Proc. of SPIE Vol. $6745674511-1$ 
straightforward comparison of the data from the system to be validated with the data from a validating system which samples exactly the same atmospheric state, and has identical characterization but negligible errors. Unfortunately, on many occasions this approach is not feasible. As a rule, the systems have different characteristics, non-negligible noise, and perform their measurements at, maybe, close but different times and locations. This type of measurements we will call correlative measurements.

It has been demonstrated ${ }^{7}$ that even when two different systems perform the measurements on the same state of the atmosphere, sensible comparison can not be reduced to simple straightforward point-by-point analysis of differences Proper statistical methods should be used instead. In practice, the situation is more complex and two factors must be taken into account: (i) the systems perform their measurements at different time and location; (ii) the systems have different characteristics, i. e. they sample the atmosphere differently on a vertical and horizontal scales. Both factors cause additional error and must be accounted for. The error caused by the first factor we will call state non-coincidence error; it is caused by atmospheric spatial nonuniformity and temporal variation. The error caused the second factor we will call characteristic difference error and it is associated with the difference between measurement systems hardware and processing.

The goal of this work is twofold: (i) Develop a theoretical model and practical technique for validation of satellite sounders by correlative measurements; the model takes into account state non-coincidence error, characteristic difference error as well as finite accuracy and precision of the validating system (Sections 1 through 4); (ii) Demonstrate practicability of the technique on real data (Section 5). We will mostly follow the terminology and notations used by Rodgers $^{2,7,8,9}$. In particular in equations, bold lower case symbols denote column vectors, e. g. $\mathbf{x}$, upper case bold typeface is used for matrices, e. g. $\mathbf{S}$, and regular italicized font is reserved for scalars.

\section{MODEL FOR MEASUREMENTS}

For the retrieved profile $\hat{\mathbf{y}}$ in linear approximation we can write ${ }^{2}$

$$
\hat{\mathbf{y}}=\mathbf{x}_{\mathrm{a}}+\mathbf{A}\left(\mathbf{x}-\mathbf{x}_{\mathrm{a}}\right)+\varepsilon_{\mathbf{y}}
$$

where $\mathbf{x}$ and $\hat{\mathbf{y}}$ are true and retrieved profiles; the averaging kernel matrix $\mathbf{A}=\frac{\partial \hat{\mathbf{y}}}{\partial \mathbf{x}}$ shows how the true state contributes to the measurement; $\mathbf{x}_{\mathbf{a}}$ is a priori profile used as linearization point; $\boldsymbol{\varepsilon}_{\mathbf{y}}$ is retrieval error. It is pertinent to point that the equation (2.1) can formally be used to describe in situ measurements, e. g. radiosonde; in this case $\mathbf{x}_{\mathbf{a}}=\mathbf{0}$ and for the most of remote sensing applications one can assume $\mathbf{A}=\mathbf{I}$, where I is identity matrix.

Mean value of the measurements is as follows:

$$
\overline{\hat{\mathbf{y}}}=\mathbf{x}_{\mathrm{a}}+\mathbf{A}\left(\overline{\mathbf{x}}-\mathbf{x}_{\mathrm{a}}\right)+\Delta_{\mathrm{y}}=\mathbf{x}_{\mathrm{a}}+\mathbf{A} \overline{\boldsymbol{\varepsilon}}_{\mathrm{x}}+\Delta_{\mathrm{y}}
$$

where $\overline{\boldsymbol{\varepsilon}}_{\mathbf{x}}=\overline{\mathbf{x}}-\mathbf{x}_{\mathbf{a}}$ and may be interpreted as an accuracy of the knowledge of the true mean value $\overline{\mathbf{x}}$ or state true mean error; the equations for the measurements can be writhen using the deviation from the true mean $\boldsymbol{\delta} \mathbf{x}$ :

$$
\hat{\mathbf{y}}=\mathbf{x}_{\mathrm{a}}+\mathbf{A} \boldsymbol{\delta} \mathbf{x}+\mathbf{A} \overline{\boldsymbol{\varepsilon}}_{\mathrm{x}}+\boldsymbol{\varepsilon}_{\mathrm{y}}
$$

where $\boldsymbol{\delta} \mathbf{x}=\mathbf{x}-\overline{\mathbf{x}}$. It is pertinent to note that in linear approximation for the retrieval $\overline{\hat{\mathbf{y}}}=\mathbf{x}_{\mathrm{a}}+\mathbf{A} \overline{\boldsymbol{\varepsilon}}_{\mathbf{x}}+\boldsymbol{\Delta}_{\mathbf{x}} \neq \overline{\mathbf{x}}+\boldsymbol{\Delta}_{\mathbf{x}}$. In other words, if $\mathbf{A} \neq \mathbf{I}$ then even with the absence of bias the mean value of the retrievals is not necessarily equal to the mean value of the true state ${ }^{2}$.

\section{CORRELATION MODEL}

In this section we will analyze the statistical correlation between two ensembles of true atmospheric states in the case when they are separated in time and space, as well as correlation between the ensembles of measurements in the case when they are performed on the same state but the measurement systems have different characteristics. 


\subsection{State non-coincidence error and correlation between true states.}

Consider the first system (the system to be validated) performing its measurement on the ensemble of true states $\mathbf{X}_{1}$

$$
\mathbf{x}_{\mathbf{1}}=\mathbf{x}(\mathbf{z}, t) \quad \mathbf{z} \in \mathbf{Z}_{\mathbf{1}} ; t \in\left[t_{1}, t_{2}\right]
$$

where $\mathbf{z}, t$ are coordinates and time. The second system (correlative system) operates on the ensemble $\mathbf{X}_{\mathbf{2}}$ and performs correlative measurements $\mathbf{x}_{2}$ separated from $\mathbf{x}_{1}$ by vector $\mathbf{d}$ and time interval $\tau$

$$
\mathbf{x}_{2}=\mathbf{x}(\mathbf{z}+\mathbf{d}, t+\tau) \quad \mathbf{z} \in \mathbf{Z}_{1} ; t \in\left[t_{1}, t_{2}\right]
$$

True mean value of the ensemble $\mathbf{X}_{1}$ is

$$
\overline{\mathbf{x}}_{1}=E\left\{\mathbf{x}_{1}\right\}=E\{\mathbf{x}(\mathbf{z}, t)\}
$$

Auto-covariance of the ensemble $\mathbf{X}_{1}$ is

$$
\mathbf{S}_{\mathbf{x}_{1}}=E\left\{\left(\mathbf{x}_{\mathbf{1}}-\overline{\mathbf{x}}_{\mathbf{1}}\right)\left(\mathbf{x}_{\mathbf{1}}-\overline{\mathbf{x}}_{\mathbf{1}}\right)^{T}\right\}
$$

For the ensemble $\mathbf{X}_{\mathbf{2}}$ true mean value is

$$
\overline{\mathbf{x}}_{2}=E\left\{\mathbf{x}_{2}\right\}=E\{\mathbf{x}(\mathbf{z}+\mathbf{d}, t+\tau)\}
$$

with corresponding auto-covariance

$$
\mathbf{S}_{\mathbf{x}_{\mathbf{2}}}=E\left\{\left(\mathbf{x}_{\mathbf{2}}-\overline{\mathbf{x}}_{\mathbf{2}}\right)\left(\mathbf{x}_{\mathbf{2}}-\overline{\mathbf{x}}_{\mathbf{2}}\right)^{T}\right\} \text {. }
$$

Correlation between the true states $\mathbf{x}_{1}$ and $\mathbf{x}_{2}$ can be characterized by cross-covariances

$$
\begin{aligned}
& \mathbf{S}_{\mathbf{1 2}}=E\left\{\left(\mathbf{x}_{\mathbf{1}}-\overline{\mathbf{x}}_{\mathbf{1}}\right)\left(\mathbf{x}_{\mathbf{2}}-\overline{\mathbf{x}}_{\mathbf{2}}\right)^{T}\right\}=E\left\{\left(\mathbf{x}(\mathbf{z}, t)-\overline{\mathbf{x}}_{1}\right)\left(\mathbf{x}(\mathbf{z}+\mathbf{d}, t+\tau)-\overline{\mathbf{x}}_{\mathbf{2}}\right)^{T}\right\} \\
& \mathbf{S}_{\mathbf{2 1}}=E\left\{\left(\mathbf{x}_{\mathbf{2}}-\overline{\mathbf{x}}_{\mathbf{2}}\right)\left(\mathbf{x}_{\mathbf{1}}-\overline{\mathbf{x}}_{\mathbf{1}}\right)^{T}\right\}=E\left\{\left(\mathbf{x}(\mathbf{z}+\mathbf{d}, t+\tau)-\overline{\mathbf{x}}_{\mathbf{2}}\right)\left(\mathbf{x}(\mathbf{z}, t)-\overline{\mathbf{x}}_{\mathbf{1}}\right)^{T}\right\}
\end{aligned}
$$

For the cross-covariances the following relations are true $\mathbf{S}_{\mathbf{1 2}}=\mathbf{S}_{\mathbf{2 1}}^{\mathbf{T}}$ and $\mathbf{S}_{\mathbf{1 2}}=\mathbf{S}_{\mathbf{2 1}}=\mathbf{S}_{\mathbf{x}_{\mathbf{1}}}=\mathbf{S}_{\mathbf{x}_{\mathbf{2}}}$ when $\tau=0$ and $\mathbf{d}=\mathbf{0}$.

Because correlation only measures linear relationship, in the following consideration we assume that the variation of the true states about their means $\overline{\mathbf{x}}_{\mathbf{1}}$ and $\overline{\mathbf{x}}_{\mathbf{2}}-\boldsymbol{\delta} \mathbf{x}_{\mathbf{1}}$ and $\boldsymbol{\delta} \mathbf{x}_{\mathbf{2}}$ are correlated so that

$$
\boldsymbol{\delta} \mathbf{x}_{1}=\mathbf{B}_{\mathbf{x}} \boldsymbol{\delta} \mathbf{x}_{2}+\boldsymbol{\xi}_{\mathbf{x}}
$$

where $\xi_{\mathbf{x}}$ is random with $\bar{\xi}_{\mathbf{x}}=0$ and covariance $\mathbf{S}_{\boldsymbol{\xi} \mathbf{x}} ; \mathbf{B}_{\mathbf{x}}$ is state correlation matrix. The relation (3.1) can also be interpreted in terms of conditional probability density function ( $p d f) P\left(\boldsymbol{\delta} \mathbf{x}_{1} \mid \boldsymbol{\delta} \mathbf{x}_{\mathbf{2}}\right)$ of $\boldsymbol{\delta} \mathbf{x}_{\mathbf{1}}$ given $\boldsymbol{\delta} \mathbf{x}_{2}$, i.e. $\mathbf{B}_{\mathbf{x}} \boldsymbol{\delta} \mathbf{x}_{2}$ is expected value of $\boldsymbol{\delta} \mathbf{x}_{1}$ given $\boldsymbol{\delta} \mathbf{x}_{2}$

$$
\mathbf{B}_{\mathbf{x}} \mathbf{\delta} \mathbf{x}_{2}=\int \delta \mathbf{x}_{\mathbf{1}} P\left(\boldsymbol{\delta} \mathbf{x}_{\mathbf{1}} \mid \boldsymbol{\delta} \mathbf{x}_{\mathbf{2}}\right) d\left(\boldsymbol{\delta} \mathbf{x}_{\mathbf{1}}\right)
$$

The term $\xi_{\mathbf{x}}$ can be interpreted as random state non-coincidence error.

For covariances $\mathbf{S}_{\mathrm{x}_{1}}, \mathbf{S}_{\mathrm{x}_{2}}$, and $\mathbf{S}_{\xi}$ the following relations are true:

$$
\begin{aligned}
& \operatorname{cov}\left(x_{2}, \xi_{x}\right)=0 \\
& S_{x_{1}}=B_{x} S_{x_{2}} B_{x}^{T}+S_{\xi x} \\
& S_{12}=B_{x} S_{x_{2}} \\
& B_{x}=S_{12} S_{x_{2}}^{-1}
\end{aligned}
$$

The matrices $\mathbf{S}_{\mathbf{1 2}}, \mathbf{B}_{\mathbf{x}}$ and $\mathbf{S}_{\xi \mathrm{x}}$ are some functions of $\mathbf{d}$ and $\tau: \mathbf{S}_{\mathbf{1 2}}=\mathbf{S}_{\mathbf{1 2}}(\mathbf{d}, \tau), \mathbf{B}_{\mathbf{x}}=\mathbf{B}_{\mathbf{x}}(\mathbf{d}, \tau), \mathbf{S}_{\xi \mathbf{x}}=\mathbf{S}_{\xi \mathbf{x}}(\mathbf{d}, \tau)$ 
The following relations are true:

$\mathbf{B}_{\mathbf{x}}(\mathbf{0}, 0)=\mathbf{I}$ and $\mathbf{S}_{\xi \mathbf{x}}(\mathbf{0}, 0)=\mathbf{0}$ and $\mathbf{B}_{\mathbf{x}}=\mathbf{0}$ when $\mathbf{S}_{\mathbf{1 2}}=\mathbf{S}_{\mathbf{2 1}}=\mathbf{0}$.

Considering the relations given by formulas (3.1) - (3.6) we can interpret the correlated term $\mathbf{B}_{\mathbf{x}} \boldsymbol{\delta} \mathbf{x}_{2}$ in Equation (3.1) as the best estimate of the state $\boldsymbol{\delta} \mathbf{x}_{1}$ which can be obtained based on the knowledge of $\boldsymbol{\delta} \mathbf{x}_{2}$. Uncorrelated term $\boldsymbol{\xi}_{\mathbf{x}}$ describes non-coincidence error of the estimate.

In practice, the matrix $\mathbf{S}_{\mathbf{x}_{2}}$ may be singular, i. e. its rank $p$ is less than its size $m_{2}$. In that case the pseudo-inverse can be used $^{7}$

$$
\tilde{\mathbf{S}}_{\mathbf{x}_{2}}^{-1}=\tilde{\mathbf{L}} \tilde{\mathbf{\Lambda}}^{-1} \tilde{\mathbf{L}}^{\mathbf{T}}
$$

where $\tilde{\mathbf{\Lambda}}$ is a $p \times p$ diagonal matrix of $p$ non-zero eigenvalues and $\tilde{\mathbf{L}}$ is the corresponding $m_{2} \times p$ matrix of eigenvectors.

\subsection{Characteristic difference error and correlation between measurements.}

Addressing the problem of comparison of measured signals Rodgers ${ }^{7}$ indicated that different instruments typically measure different physical quantities, have different weighting functions and, moreover, it may happen that they do not measure any subspace of state space in common. To resolve this issue he analyzed linear combinations of the weighting functions that are similar enough for useful comparison. In the current work we will use the correlative approach, the same one as we used for the analysis of the relation between true states.

Measurements of the same state by two systems are described by the following equations:

$$
\begin{aligned}
& \delta y_{1}=A_{1} \delta x+A_{1} \bar{\varepsilon}_{x}+\varepsilon_{1 y} \\
& \delta y_{2}=A_{2} \delta x+A_{2} \bar{\varepsilon}_{x}+\varepsilon_{2 y}
\end{aligned}
$$

where

$$
\begin{aligned}
& \delta y_{1}=\hat{y}_{1}-x_{a} \\
& \delta y_{2}=\hat{y}_{2}-x_{a} \\
& \bar{\varepsilon}_{x}=\bar{x}-x_{a}
\end{aligned}
$$

The terms $\mathbf{A}_{\mathbf{1}} \overline{\boldsymbol{\varepsilon}}_{\mathbf{x}}$ and $\mathbf{A}_{\mathbf{2}} \overline{\boldsymbol{\varepsilon}}_{\mathbf{x}}$ are systematic errors caused by limited knowledge of the true mean of the ensemble $\mathbf{X}$.

Analogously to the relation between true states we describe the relation between measurements in the form of the sum of correlated and uncorrelated terms:

$$
\mathbf{A}_{1} \delta \mathbf{x}=\mathbf{B}_{\mathbf{y}} \mathbf{A}_{2} \delta \mathbf{x}+\xi_{\mathbf{y}}
$$

The term $\xi_{\mathbf{y}}$ can be interpreted as random characteristic difference error; $\mathbf{B}_{\mathbf{y}}$ is measurement correlation matrix. For the measurement auto-covariance we have the relation:

$$
E\left\{\delta \mathbf{y}_{1} \delta \mathbf{y}_{1}^{\mathrm{T}}\right\}=\mathbf{A}_{1} \mathbf{S}_{\mathbf{x}} \mathbf{A}_{1}^{\mathrm{T}}=\left(\mathbf{B}_{\mathbf{y}} \mathbf{A}_{2}\right) \mathbf{S}_{\mathbf{x}}\left(\mathbf{B}_{\mathbf{y}} \mathbf{A}_{2}\right)^{\mathrm{T}}+\mathbf{S}_{\xi \mathbf{y}}
$$

and for the measurement cross-covariance

$$
E\left\{\boldsymbol{\delta} \mathbf{y}_{1} \delta \mathbf{y}_{2}^{\mathbf{T}}\right\}=\mathbf{A}_{1} \mathbf{S}_{\mathbf{x}} \mathbf{A}_{\mathbf{2}}^{\mathbf{T}}=\mathbf{B}_{\mathbf{y}}\left(\mathbf{A}_{\mathbf{2}} \mathbf{S}_{\mathbf{x}} \mathbf{A}_{\mathbf{2}}^{\mathbf{T}}\right)
$$

hence

$$
B_{y}=A_{1} S_{x} A_{2}^{T}\left(A_{2} S_{x} A_{2}^{T}\right)^{-1}
$$

The solution only exists if the matrix $\left(\mathbf{A}_{2} \mathbf{S}_{\mathbf{x}} \mathbf{A}_{2}^{\mathbf{T}}\right)$ is nonsingular. In case of its singularity, likewise in equation (3.7), the pseudo-inverse can be used:

$$
\tilde{\mathbf{S}}^{-1}=\tilde{\mathbf{L}} \tilde{\mathbf{\Lambda}}^{-1} \tilde{\mathbf{L}}^{\mathbf{T}}
$$


where $\tilde{\mathbf{\Lambda}}$ and $\tilde{\mathbf{L}}$ have the same meaning as in equation (3.7) but for the matrix $\left(\mathbf{A}_{2} \mathbf{S}_{\mathbf{x}} \mathbf{A}_{2}^{\mathbf{T}}\right)$.

\section{MODEL FOR VALIDATION}

In this section using correlative model from the previous chapter we will derive the equations for statistical characteristics of the validation error as well as practical formulas for their estimate based on sample measurements.

The correlative measurements performed by two systems can be described by the following equations:

$$
\begin{aligned}
& \delta y_{1}=\hat{y}_{1}-x_{1 a}=A_{1}\left(x_{1}-x_{1 a}\right)+\varepsilon_{1 y} \\
& \delta y_{2}=\hat{y}_{2}-x_{2 a}=A_{2}\left(x_{2}-x_{2 a}\right)+\varepsilon_{2 y}
\end{aligned}
$$

The target parameter of the present study is the error of the validated (the first) system.

$$
\varepsilon_{1 \mathrm{y}}=\delta y_{1}-A_{1}\left(x_{1}-x_{1 a}\right)
$$

In the process of validation we need to estimate $\mathbf{A}_{1}\left(\mathbf{x}_{1}-\mathbf{x}_{1 \mathrm{a}}\right)$ based on correlative measurements.

Let's present the term $\mathbf{A}_{\mathbf{1}}\left(\mathbf{x}_{1}-\mathbf{x}_{1 \mathrm{a}}\right)$ in the form

$$
A_{1}\left(x_{1}-x_{1 a}\right)=\overline{A_{1}\left(x_{1}-x_{1 a}\right)}+\varepsilon_{v a l}
$$

where $\boldsymbol{\varepsilon}_{\text {val }}$ is validation error; then the estimate of the error of validated system $\hat{\boldsymbol{\varepsilon}}_{\mathbf{1 y}}$ is

$$
\hat{\varepsilon}_{1 y}=\delta y_{1}-\overline{A_{1}\left(x_{1}-x_{1 a}\right)}=\varepsilon_{1 y}+\varepsilon_{\text {val }}
$$

Our goals are development of practical technique for inferring of $\overline{\mathbf{A}_{1}\left(\mathbf{x}_{1}-\mathbf{x}_{1 \mathbf{a}}\right)}$ from correlative measurements and estimation of the validation error and its statistical characteristics.

Analogously to equation (3.2) we interpret equation (3.10)

$$
\mathbf{B}_{\mathbf{y}} \mathbf{A}_{2} \delta \mathbf{x}_{2}=\int \mathbf{A}_{1} \delta \mathbf{x}_{1} P\left(\mathbf{A}_{1} \delta \mathbf{x}_{1} \mid \mathbf{A}_{2} \delta \mathbf{x}_{2}\right) d\left(\mathbf{A}_{1} \delta \mathbf{x}_{1}\right)
$$

where $P\left(\mathbf{A}_{\mathbf{1}} \boldsymbol{\delta} \mathbf{x}_{\mathbf{1}} \mid \mathbf{A}_{\mathbf{2}} \boldsymbol{\delta} \mathbf{x}_{\mathbf{2}}\right)$ is the conditional probability density function of $\mathbf{A}_{\mathbf{1}} \boldsymbol{\delta} \mathbf{x}_{\mathbf{1}}$ given $\mathbf{A}_{\mathbf{2}} \boldsymbol{\delta} \mathbf{x}_{\mathbf{2}}$. Hence $\mathbf{B}_{\mathbf{y}} \boldsymbol{\delta} \mathbf{y}_{\mathbf{2}}$ can be taken for the best estimate of $\mathbf{A}_{1}\left(\mathbf{x}_{1}-\mathbf{x}_{1 \mathbf{a}}\right)$ in a sense of expected value inferred from correlative measurements

$$
\overline{A_{1}\left(x_{1}-x_{1 a}\right)}=B_{y} \delta y_{2}
$$

Thus the linear model for the validation by correlative measurements is

$$
\hat{\varepsilon}_{1 \mathrm{y}}=\delta y_{1}-B_{\mathrm{y}} \delta y_{2}=\varepsilon_{1 \mathrm{y}}+\varepsilon_{\mathrm{val}}
$$

The validation error is

$$
\varepsilon_{\text {val }}=A_{1}\left(x_{1}-x_{1 a}\right)-\overline{A_{1}\left(x_{1}-x_{1 a}\right)}=A_{1}\left(x_{1}-x_{1 a}\right)-B_{y} \delta y_{2}
$$

Using relations (3.1), (3.8), (3.9), (3.10), (4.1), and (4.2) we can rewrite it

$$
\begin{aligned}
\boldsymbol{\varepsilon}_{\mathrm{val}} & =\mathbf{A}_{1} \xi_{\mathrm{x}}+\xi_{\mathrm{xy}}-\mathbf{B}_{\mathrm{y}} \boldsymbol{\varepsilon}_{2 \mathrm{y}} & & \text { random } \\
& +\mathbf{A}_{1} \overline{\boldsymbol{\varepsilon}}_{1 \mathrm{x}}-\mathbf{B}_{\mathrm{y}} \mathbf{A}_{2} \overline{\boldsymbol{\varepsilon}}_{2 \mathrm{x}} & & \text { systematic }
\end{aligned}
$$

where the characteristic difference error and measurement correlation matrix are calculated as follows:

$$
\begin{aligned}
& B_{y}=A_{1} S_{12} A_{2}^{T}\left(A_{2} S_{x} A_{2}^{T}\right)^{-1} \\
& \xi_{x y}=\left(A_{1} B_{x}-B_{y} A_{2}\right) \delta x_{2} \\
& S_{\xi x y}=\left(A_{1} B_{x}-B_{y} A_{2}\right) S_{x_{2}}\left(A_{1} B_{x}-B_{y} A_{2}\right)^{T}
\end{aligned}
$$


Thus we see that three independent terms contribute to the random component of the validation error, i. e. state noncoincidence error $-\mathbf{A}_{1} \xi_{\mathbf{x}}$, combined state non-coincidence and characteristic difference error $-\xi_{\mathrm{xy}}$, and measurement error of the correlative system $-\mathbf{B}_{\mathbf{y}} \boldsymbol{\varepsilon}_{\mathbf{2}}$.

Covariance of the random component of the validation error is

$$
S_{v a l}=S_{\xi x y}+A_{1} S_{\xi x} A_{1}^{T}+B_{y} S_{2 \varepsilon y} B_{y}^{T}
$$

The mean value of the validation error is

$$
\bar{\varepsilon}_{\text {val }}=A_{1} \bar{\varepsilon}_{1 x}-B_{y}\left(A_{2} \bar{\varepsilon}_{2 x}+\Delta_{2}\right)
$$

where $\boldsymbol{\Delta}_{\mathbf{2}}=E\left\{\boldsymbol{\varepsilon}_{2 \mathrm{y}}\right\}$ is bias in the correlative measurements.

The mean value of the validation error sets the limit for the attainable accuracy of the validation. If in the process of validation a sample of $N$ measurements is taken then the estimate of the bias of the validated system is the sample mean:

$$
\hat{\Delta}_{1}=\frac{1}{N} \sum_{i=1}^{N}\left(\boldsymbol{\delta} \mathbf{y}_{1 i}-\mathbf{B}_{\mathbf{y}} \boldsymbol{\delta} \mathbf{y}_{2 i}\right)=\Delta_{1}+\bar{\varepsilon}_{\mathrm{val}}+\boldsymbol{\varepsilon}_{1 \Delta}
$$

where $\boldsymbol{\varepsilon}_{\mathbf{1}}$ is error of the sample mean with covariance

$$
\mathbf{S}_{1 \Delta}=\frac{1}{N}\left(\mathbf{S}_{1 \mathrm{ky}}+\mathbf{S}_{\mathrm{val}}\right)
$$

Unbiased estimate of the error covariance is sample covariance about sample mean

$$
\hat{\mathbf{S}}=\frac{N}{N-1} \sum_{i=1}^{N}\left[\hat{\boldsymbol{\Delta}}_{\mathbf{1}}-\left(\boldsymbol{\delta} \mathbf{y}_{\mathbf{1 i}}-\mathbf{B}_{\mathbf{y}} \boldsymbol{\delta} \mathbf{y}_{\mathbf{2} i}\right)\right]\left[\hat{\boldsymbol{\Delta}}_{\mathbf{1}}-\left(\boldsymbol{\delta} \mathbf{y}_{\mathbf{1} i}-\mathbf{B}_{\mathbf{y}} \boldsymbol{\delta} \mathbf{y}_{\mathbf{2}}\right)\right]^{\mathbf{T}}
$$

and the estimate of the covariance of the measurement error of the validated system $\hat{\mathbf{S}}_{1 \mathrm{y} y}$ can be found from the following:

$$
\hat{\mathbf{S}}_{1 \mathrm{\varepsilon y}}=\hat{\mathbf{S}}-\mathbf{S}_{\text {val }}
$$

The equations (4.13), (4.15) and (4.16) give the practical recipe for validation by correlative measurements. For practical application one needs the measurement correlation matrix $\mathbf{B}_{\mathbf{y}}$ and a priori statistical characteristics of the validation error, i. e. $\overline{\boldsymbol{\varepsilon}}_{\text {val }}$ and $\mathbf{S}_{\text {val }}$. They can be estimated using equations (4.11), (4.12), and (4.13) with associated formulas, and a priori statistical characteristics of the true state ensembles. In the following section we will give an example of practical implementation of the developed validation model.

\section{CASE STUDY}

\subsection{State Non-coincidence Error.}

We will estimate the state non-coincidence $\operatorname{error} \xi_{\mathrm{x}}(\tau)$ as a function of time difference $\tau$ for three launch sites, i. e Tropical Western Pacific (TWP; 0.52 ${ }^{\circ} \mathrm{S}, 166.92^{\circ} \mathrm{E}$ ), Southern Great Planes (SGP; $36.37^{\circ} \mathrm{N}, 97.30^{\circ} \mathrm{W}$ ) ARM sites, and Lindenberg (LND; $\left.52.21^{\circ} \mathrm{N}, 14.12^{\circ} \mathrm{E}\right)$ station (Germany). The launch schedules at those stations are different. At the SGP site some dedicated launches have been made $^{3}$ so we could examine the cases when time difference was between 20 min and 1 hour (corresponds to average $\tau=40 \mathrm{~min}$ ), as well as $\tau=3,6,12,24,48$, and 96 hours. At the LND site sondes are launched every 6 hours and at the TWP site every 12 hours. In the following consideration we assume that the radiosonde profiles accurately represent the true state.

For each station the data were de-seasonalized and then from the whole set of available profiles for a particular $\tau$ we constructed two ensembles such that each sonde in the first ensemble $\mathbf{X}_{\mathbf{1}}$ had at least one reciprocal sonde in the second ensemble $\mathbf{X}_{2}$ with the launch time difference $\tau$. For those ensembles we calculated auto- and cross- 
covariances $\mathbf{S}_{\mathbf{x}_{1}}, \mathbf{S}_{\mathbf{x}_{2}}, \mathbf{S}_{\mathbf{1 2}}(\tau)$ and, subsequently, using equations (3.4) - (3.7), the covariance of the state noncoincidence error $-\mathbf{S}_{\xi \mathbf{x}}(\tau)$.

Some results of the calculations are presented in the Figures 1 - 3. The color panels in Figure 1 illustrate the magnitude of the variations and the off-diagonal structure illustrates the correlation between levels.

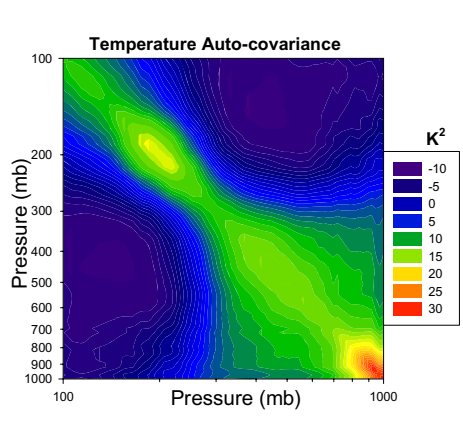

Temperature State Non-coincidence Error

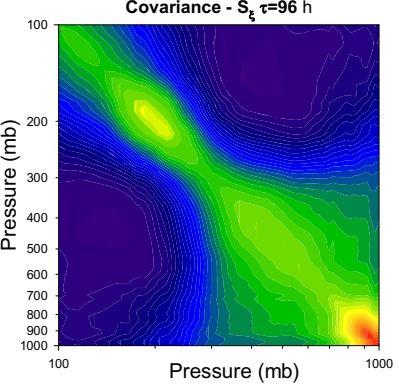

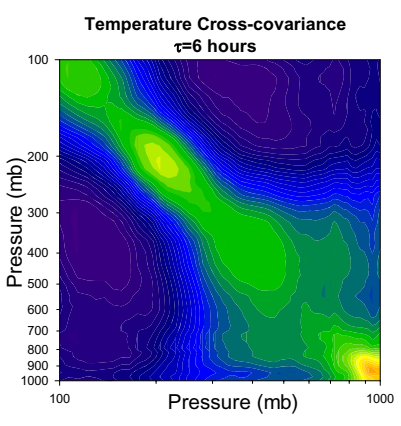
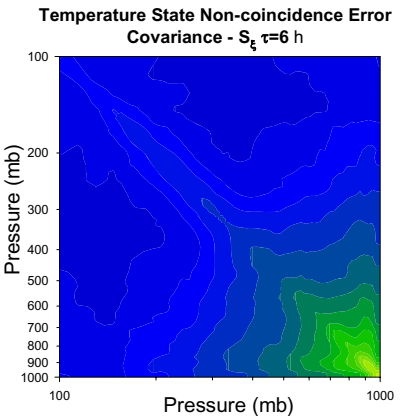
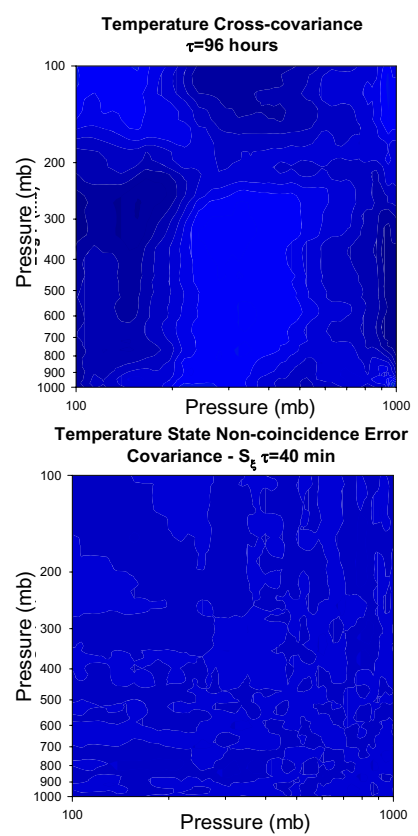

Fig. 1 Covariance matrices for temperature variation, correlation, and state non-coincidence error calculated for SGP site data set ${ }^{3}$

Comparing $\mathbf{S}_{\xi \mathbf{x}}(\tau=96 h)$ with auto-covariance one can see that at the SGP site in four days the atmosphere almost completely "forgets" its state. In this case validation by correlative measurements becomes validation against a priori, e.g. in ref. 6. In the Figure 2 the square root of the diagonal elements of error covariance $\mathbf{S}_{\xi \mathbf{x}}(\tau)$ are plotted.

The rms error can be used as a simple quantitative metric for the state non-coincidence error. It would be natural to expect that spatial and temporal characteristics of the state non-coincidence error are different at different climate zones. The considered sites represent tropics (TWP), continental climate (SGP), and temperate mid-latitude climate (LND). To illustrate the differences the rms error for all three considered sites and for the most interesting for validation time intervals $\tau \leq 12$ hours are presented in the Figure 2. In the Figure 3 we present more aggregated characteristic illustrating temporal dependence of the $r m s$ in the free troposphere $(800-300 \mathrm{mb}$ ), and in the boundary layer (surface $800 \mathrm{mb})$.

One can see that at SGP site the $r m s$ error exhibits almost linear behavior when $\tau \leq 6 \mathrm{~h}$ with a slope $0.42 \mathrm{~K} h^{-1}$ in free troposphere and $0.63 \mathrm{~K} \mathrm{~h}^{-1}$ in the boundary layer; the absolute value of intercept in both cases does not exceed $0.1 \mathrm{~K}$. For LND site we don't have the data with $\tau<6 \mathrm{~h}$; therefore, we make an assumption that at LND for $\tau<6 \mathrm{~h}$ the error can also be approximated linearly with zero intercept and the slope $0.24 \mathrm{~K}^{-1}$ in free troposphere and $0.32 \mathrm{~K} h^{-1}$ in the boundary layer. 


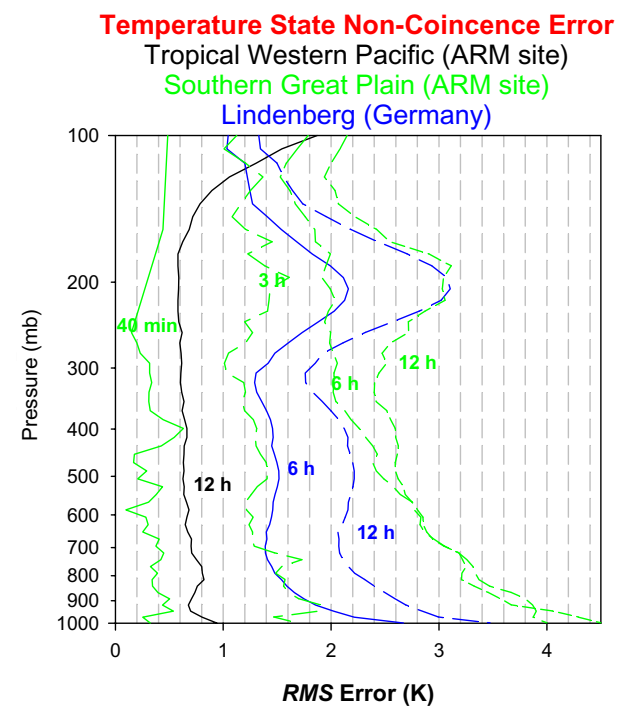

Fig. 2. The rms error for three different sites as a function of altitude. Color indicates the site: black is for TWP, green is for SGP, and blue is for LND. The numbers by the curve denote the corresponding time difference.

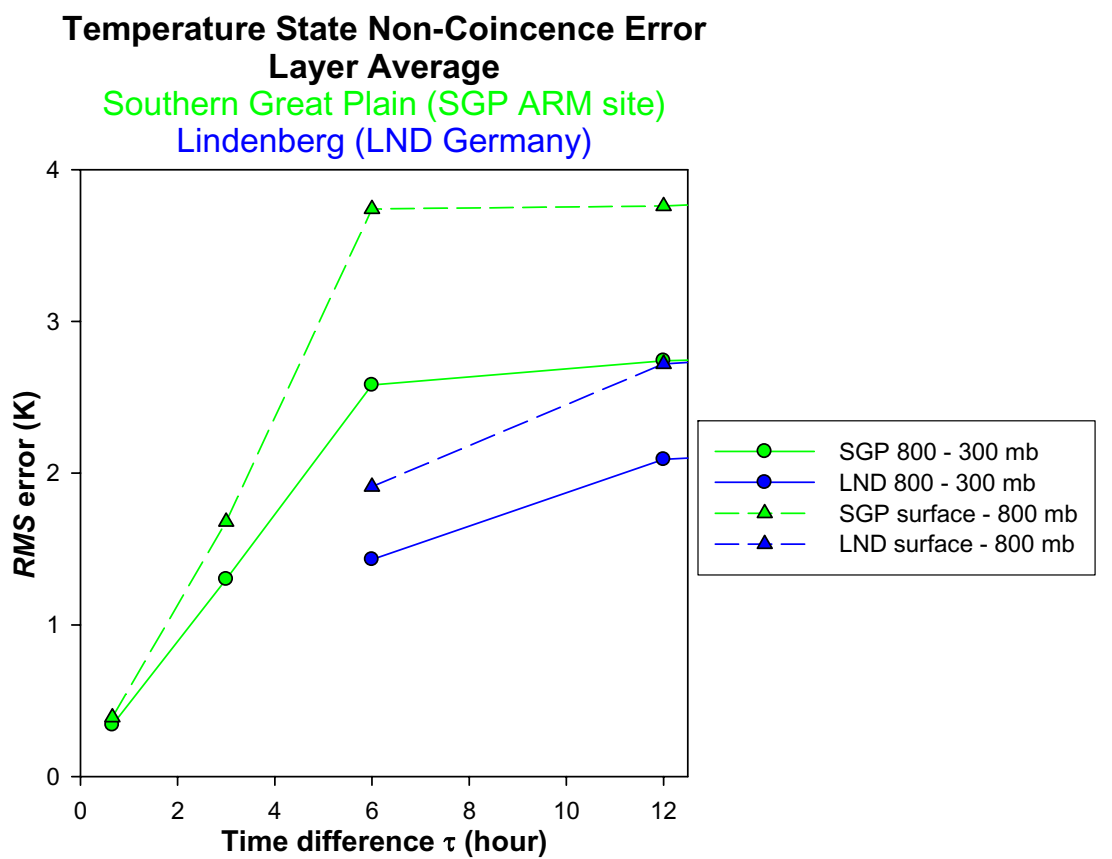

Fig. 3. The rms error averaged in layers: triangles and dashed lines are for mid and upper troposphere ( $800-300 \mathrm{mb})$; circles and solid lines are for boundary layer (surface $-800 \mathrm{mb}$ ). Color indicates the site: green is for SGP, blue is for LND.

\subsection{Validation Error.}

Three factors contribute to the random component of the total validation error (see equation (4.10)), i. e. state noncoincidence, instrument difference and measurement noise. In the current study we neglect the smoothing of the true temperature profile by radiosonde. Then the equations (3.8) and (3.9) for measurements take the form

$$
\begin{aligned}
& \delta y_{1}=\mathbf{A} \delta x+A \bar{\varepsilon}_{x}+\varepsilon_{1 y} \\
& \delta y_{2}=\delta x+\bar{\varepsilon}_{x}+\varepsilon_{2 y}
\end{aligned}
$$


where $\mathbf{y}_{\mathbf{1}}$ and $\mathbf{y}_{\mathbf{2}}$ have the meaning of measured temperature profiles; $\mathbf{A}$ is averaging kernel matrix for the sounder. In this case the instrument correlation matrix is $\mathbf{B}_{\mathbf{y}}=\mathbf{A}$ and the characteristic difference error $\boldsymbol{\xi}_{\mathbf{x y}}=\mathbf{0}$. Without accounting for radiosonde noise the random validation error caused by state non-coincidence is $\boldsymbol{\varepsilon}_{\mathrm{val}}=\mathbf{A} \boldsymbol{\xi}_{\mathrm{x}}$ with covariance $\mathbf{S}_{\mathrm{val}}=\mathbf{A} \mathbf{S}_{\xi \mathbf{x}} \mathbf{A}^{\mathbf{T}}$ where $\mathbf{S}_{\xi \mathrm{x}}$ is derived from equations (3.4)- (3.6).

The Figure 4 presents the covariance matrices of the validation error and corresponding state non-coincidence error caused by time difference $\tau=3$ hours. The rms errors are presented in the Figure 5. Averaging kernels typical for atmospheric sounders such as AIRS and IASI were taken from Pougatchev et. $\mathrm{al}^{10}$.
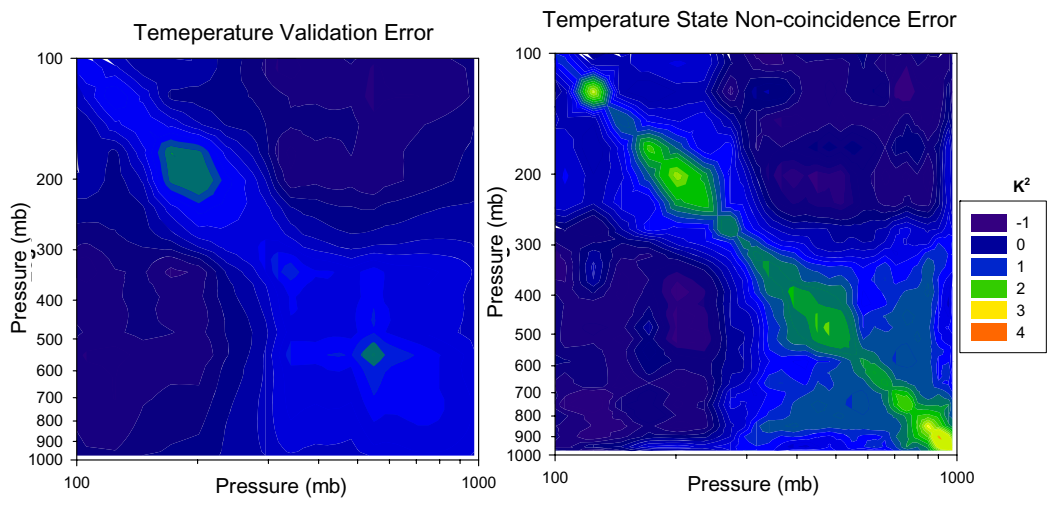

Fig. 4. Covariance matrices for temperature sounding validation error (left panel) caused by non-coincidence error (right panel) with time difference $\tau=3$ hours.

RMS Validation and State Non-coincidence Error

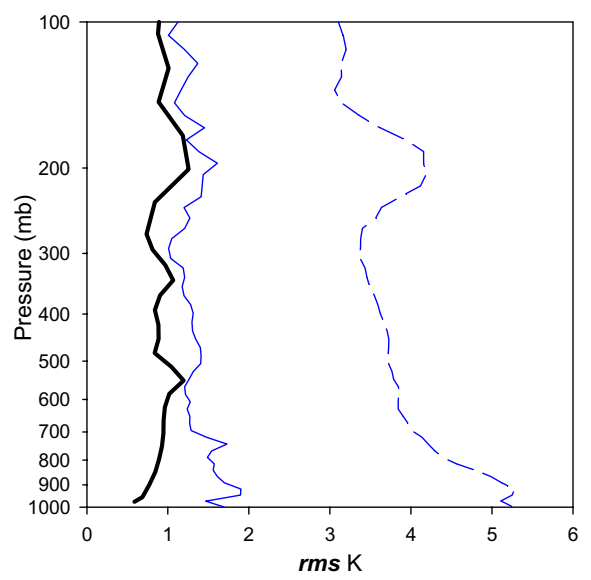

Fig. 5. RMS error for the matrices in figure 6. Black curve is validation $\mathrm{rms}$; blue solid line is corresponding $r m s$ of the state noncoincidence error; dashed blue line is the square root of diagonals of the auto-covariance matrix.

Based on the performed analysis we estimated a random component of the validation error for IASI and AIRS instrument overpasses at TWP, SGP and LND sites. The results are presented in the Figure 6. 


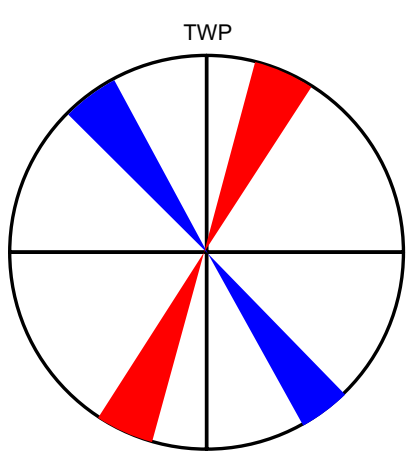

For TWP rms below $0.6 \mathrm{~K}$

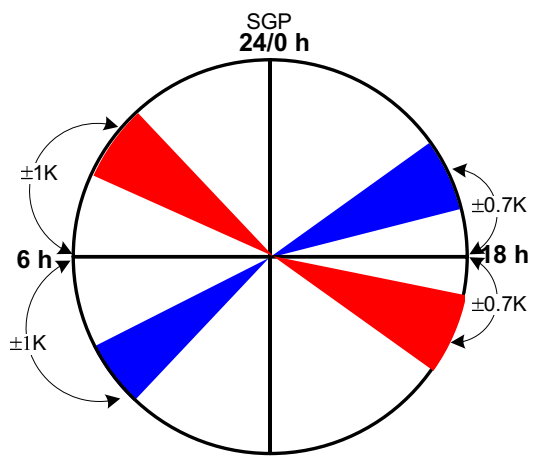

$12 \mathrm{~h}$

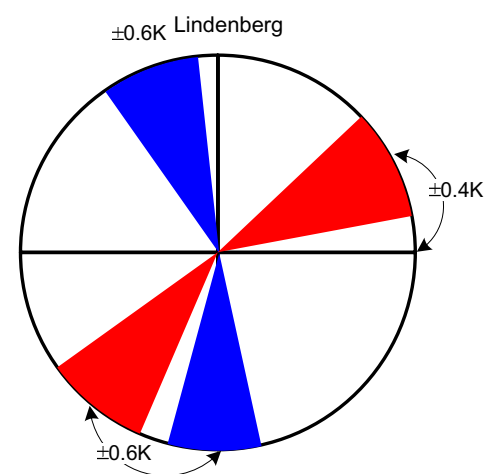

Fig. 6. Validation rms non-coincidence error for AIRS and IASI overpasses at three launch sites. Black crosshair lines corresponds to standard launch times, colored sectors shows the time intervals of overpasses: blue is for AIRS and red is for IASI.

\section{CONCLUSIONS}

In the presented work we developed the theoretical basis and practical technique which are needed to properly validate atmospheric sounders by correlative measurements with the presence of non-coincidence and instrument characteristic differences. The developed approach requires prior to validation knowledge of statistical characteristics of the atmospheric states on which the measurements are performed as well as characteristics of the systems, i. e. their averaging kernels. The technique provides the recipe for calculation of the linear estimate of the measurements of the validated sounder as well as its error. In the case study we demonstrated the practicability of the approach by applying it to real radiosonde data. The developed approach can be used for interpretation of the results of different validation activities as well as for the purposes of planning.

\section{Acknowledgments.}

This work was funded by NPOESS IPO NOAA contract DG133E-06-CQ-0079.

\section{REFERENCES}

1. GEOSS 2007, http://www.noaa.gov/eos.html

2. Rodgers, C.D., Inverse Methods for Atmospheric Sounding: Theory and Practice, World Scientific Publishing Co. Ltd., 2000.

3. D. C. Tobin, H. E. Revercomb, R. O. Knuteson, B. Lesht, L. L. Strow, S. E. Hannon, W. F. Feltz, L. Moy, E. J. Fetzer and T. Cress, "Atmospheric Radiation Measurement site atmospheric state best estimates for Atmospheric Infrared Sounder temperature and water vapor retrieval validation,” J. Geophys. Res., $\quad$ 111(D09S14), doi:10.1029/2005JD006103, 2006.

4. Migliorini, S., C. Piccolo, and C. D. Rodgers, Intercomparison of direct and indirect measurements: Michelson Interferometer for Passive Atmospheric Sounding (MIPAS) versus sonde ozone profiles, J. Geophys. Res., 109, D19316, doi:10.1029/2004JD004988, 2004.

5. Smith W. L., A. M. Larar, S. A. Mango, H. B. Howell, R. O. Knuteson, H. E. Rivercomb, and W. L. Smith Jr., The NOESS Sounding Testbed Interferometer - Remotely Sensed surface and atmospheric conditions during CLAMS., J Atmos. Sci., 62, 1117 -1133, 2005.

6. Lary D. J. and L. Lait, "Using Probability Distribution Functions for Satellite Validation, Geoscience and Remote Sensing," IEEE Transactions, 44(5), 1359 - 13662006.

7. Rodgers, C. D., and B. J. Connor, Intercomparison of remote sounding instruments, J. Geophys. Res., 108(D3), 4116, doi:10.1029/2002JD002299, 2003.

8. Rodgers, C. D., Retrieval of Atmospheric Temperature and Composition From Remote Measurements of Thermal Radiation, Rev. Geophys. and Space Phys., 14, p609-624, 1976.

9. Rodgers, C. D., Characterization and error analysis of profiles retrieved from remote sounding measurements, J. Geophys. Res., 95, 5587-5595, 1990. 
10. Pougatchev Nikita., Gail Bingham, Joel Cardon, Karen St. Germain, Stephen Mango, Joe Tansock, Vladimir Zavyalov, Stanislav Kireev, and David Tobin, Validation assessment model for atmospheric retrievals, Proc. SPIE 6301, 63010M (2006) 